\title{
Estudos Sobre EducaÇấo Matemática EM Goiás: UM OlHar SOBRE OS PROGRAMAS DE PÓS-GRADUAÇÃO STRICTO SENSU
}

\author{
Wellington L. Cedro ${ }^{1}$ \\ Aldney B. Couto ${ }^{2}$
}

LUCIANO A. HIZIM ${ }^{3}$

\section{RESUMO}

Nesta pesquisa, fizemos um mapeamento da produção científica em Educação Matemática realizada nas Instituiçôes de Ensino Superior localizadas no Estado de Goiás, no período de 1990 a 2004. O objetivo deste trabalho foi o de traçar o caminho percorrido até hoje e apresentar possíveis rumos e desafios para a comunidade de educadores matemáticos do Estado de Goiás, em direção à consolidação deste campo de conhecimento. As conclusões desta pesquisa determinam que o grande desafio da comunidade goiana de educadores matemáticos é o de, realmente, se constituir como um grupo de sujeitos atuantes em relação à produção científica, pois o panorama vislumbrado indica uma precariedade e insuficiência de trabalhos relacionados à Educação Matemática.

Palavras-ChavE: Educação Matemática, estado da arte, pesquisa em Goiás.

Studies on Mathematical Education in Goiás: one look on stricto sensu post-graduation programs

\section{ABSTRACT}

In this research we made a state-of-the-art of Mathematical Education in Goiás, during the 1990 and the 2004. The objective of this work was to trace the way covered until today and presenting possible routes and challenges for the community of mathematical educators of Goiás, in direction the consolidation of this field of knowledge. The conclusions of this research, really point that the great challenge of the local community of Mathematical Educators is presented in the form of it to consist, as a group of operating citizens in relation to the scientific production, therefore the glimpsed panorama points with respect to a precariousness and insufficiency of works related to the Mathematical Education.

KEY wORDS: mathematical education, state-of-the-art, research in Goiás.

1 Mestre em Educação (área: Ensino de Ciências e Matemática) pela FEUSP e professor do IME/UFG. E-mail: wcedro@mat.ufg.br.

2 Licenciado em Matemática pela UnUCC/UEG e professor da ETEC/Itauçu-GO.

3 Mestre em Educação pela UCG e professor da Secretaria Municipal de Educação de Goiânia-GO. E-mail: hizim@bol.com.br. 
164 Revista Soltaa Voz, v. 17, n. 2

\section{INTRODUÇÃO}

Este trabalho caracteriza-se como um "estado da arte" ou "estado do conhecimento", portanto tem caráter eminentemente bibliográfico. Neste trabalho pretendemos delinear a produção do conhecimento sobre a Educação Matemática, nas universidades situadas no Estado de Goiás, a partir de um mapeamento da produção científica na área, publicada em dissertaçôes apresentadas em programas de pós-graduação stricto sensu em Educação no período de 1990 a 2004. A escolha do período de quinze anos (1990-2004) selecionado nesta pesquisa justifica-se, pois a primeira dissertação foi defendida no ano de 1990 na Faculdade de Educação da Universidade Federal de Goiás.

O desenvolvimento deste trabalho visa a consolidação e integração da produção científica referente à Educação Matemática realizada nos programa de pós-graduação strictu sensu vinculados às universidades do Estado de Goiás, com o restante da produção nacional desta área. Desta forma estabeleceremos um processo constante de atualização das informações. Devemos esclarecer que ao enforcarmos especificamente a produção científica do Estado de Goiás, não estamos excluindo as influências nacionais e internacionais que sofreram essas obras, mas, pelo contrário estaremos situando a produção local a partir desses referenciais mais amplos. Contribuindo, assim, para o debate direcionado a solução dos problemas e das demandas regionais.

A análise aqui realizada se deu a partir das dissertações defendidas nos seguintes cursos de pós-graduação strictu sensu: mestrado em Educação Brasileira da Universidade Federal de Goiás (UFG); mestrado em Educação da Universidade Católica de Goiás (UCG) e mestrado em Ciências da Educação Superior da Universidade Estadual de Goiás (UEG) desenvolvido em convênio com a Universidade de Havana/Cuba.

\section{EDUCAÇÃO MATEMÁTICA: UM BREVE PANORAMA}

Classificada por D’Ambrósio (1993) como um ramo da Educação em que se procura estudar e desenvolver modos mais eficientes de se ensinar Matemática, a Educação Matemática, acreditamos, representar, sim, uma substanciosa oportunidade de se fazer face às dificuldades e desafios inerentes aos processos de ensino e aprendizagem em Matemática.

Trata-se de uma área eminentemente multidisciplinar que segundo D’Ambrósio (1986, p. 35), "se pratica com um objetivo geral bem especí- 
fico - transmitir conhecimentos e habilidades Matemáticas - através dos sistemas educativos (formal, não-formal e informal)".

Esse caráter multidisciplinar, inequivocamente, é o principal responsável pela vitalidade da Educação Matemática, uma vez que favorece o progresso dessa área em várias frentes de pesquisa. De fato, o estudioso e o pesquisador em Educação Matemática se vêem envolvidos com a análise dos aspectos filosóficos, epistemológicos, sociais, psicológicos e históricos relacionados ao conhecimento matemático.

O fato é que, principalmente nas últimas décadas, tem aumentado a quantidade de grupos ou linhas de pesquisa em Educação Matemática no interior dos programas de pós-graduação em Educação (Machado, Fonseca, Gomes, 2002), "alavancando" ainda mais a produção acadêmica.

Contudo, mesmo guarnecida com tantos aspectos positivos e avanços, a Educação Matemática deve, ainda assim, ser, prudentemente, considerada um campo científico em formação. Há muito que se definir, reestruturar e fundamentar nessa área, e compete a nós, estudiosos e pesquisadores, educadores matemáticos ou não, imergir em nossas reflexões e investigações de modo que possamos assistir produtivamente o processo de consolidação da Educação Matemática.

\section{O CONTEXTO GOIANO DA EDUCAÇÃO MATEMÁTICA}

A produção em Educação Matemática no Estado de Goiás tem uma história bem recente e curta, apesar de já termos cursos de Matemática funcionando há mais de quarenta anos dentro do estado.

De acordo com Silva (2003), a abertura dos primeiros cursos de graduação em Matemática (na Universidade Federal de Goiás em 1963 e na Universidade Católica de Goiás em 1961) ocorreu no início da década de 1960. Desde então, tivemos uma grande expansão de oferta de cursos de graduação em Matemática. Se nos idos dos anos 60, só tínhamos dois cursos, agora de acordo com o MEC, o Estado de Goiás possui 51 cursos, que abrangem as modalidades de bacharelado e de licenciatura (plena e parcelada), distribuídos pela capital e interior.

Apesar do grande número de cursos de graduação, a situação com relação à oferta de programas de pós-graduação é precária. O estado possui, segundo a Capes (2005), somente dois programas de pós-graduação stricto sensu que poderiam desenvolver trabalhos direcionados à Educação Matemática. 


\section{RevistaSoltaa Voz, v. 17, n. 2}

Um deles é o da Faculdade de Educação da Universidade Federal de Goiás (mestrado e doutorado em Educação Brasileira) e outro da Universidade Católica de Goiás (mestrado em Educação). Porém, nenhum destes programas, conta oficialmente em seus projetos com linhas de pesquisa destinadas à Educação Matemática ou ao ensino de Matemática. Os trabalhos publicados provenientes destes cursos surgiram de iniciativas isoladas. Isto pode ser constatado quando comparamos o número de dissertações defendidas, que podem ser enquadradas na área de Educação Matemática, em relação à quantidade total de trabalhos defendidos nestas instituiçõos até 2004. Na Faculdade de Educação da UFG somente seis trabalhos dentro de um universo de 235 dissertações defendidas (FE-UFG, 2005). Na UCG quatro dissertações dentro de um aglomerado de 69 trabalhos defendidos (UCG, 2005).

Além destes dois programas regulares, em 2001 e 2002, a UEG no intuito de qualificar parte do seu quadro docente, ofereceu um programa de mestrado interinstitucional em Ciências da Educação Superior (MINTER) em Convênio com a Universidade de Havana, no qual encontramos dois trabalhos em um conjunto de 54 pesquisas apresentadas.

\section{AS PESQUISAS ANALISADAS}

A partir do contexto descrito anteriormente, o mapeamento da produção em Educação Matemática nos programas de pós-graduação das universidades situadas no Estado de Goiás realizado por nós, conseguiu detectar um número de doze trabalhos, organizados no quadro 1, que podem ser enquadrados nesta área da produção científica.

Passemos agora a uma breve descrição das dissertaçōes defendidas, que serão apresentadas a partir do ano de defesa do trabalho.

O primeiro trabalho direcionado à Educação Matemática, defendido nos programas de pós-graduação das universidades goianas, foi o de Varizo (1990). Este estudo teve como objetivo compreender a natureza e a gênese do fazer pedagógico do professor de Matemática. Esta pesquisa de cunho etnográfico demonstrou que o fazer pedagógico dos professores pesquisados apresenta uma surpreendente semelhança no ritual pedagógico: procedimento de ensino se limitando a mostrar, repetir e praticar. Os professores escolhem os livros didáticos seguindo critérios, também, semelhantes: linguagem simples e muitos exercícios; têm uma visão fragmentada do conhecimento matemático; não estabelecem as relações verticais entre o conteúdo a ser 
ministrado na escola e os conteúdos estudados na universidade; dissociam Matemática e Geometria; não estabelecem relações entre o conhecimento matemático e o mundo; alija as disciplinas pedagógicas; não ambicionavam a profissão de educador; e, são oriundos de uma mesma camada social. Os professores entrevistados, também, não conseguem chegar a um equacionamento racional das dificuldades que enfrentam no seu dia-a-dia.

QUADRO 1: As dissertaçôes: ano, autor, título e instituição

\begin{tabular}{|c|c|c|c|}
\hline Ano & Autor & Título da dissertação & Instituição \\
\hline 1990 & $\begin{array}{l}\text { Zaíra da Cunha Melo } \\
\text { Varizo }\end{array}$ & $\begin{array}{l}\text { História de vida e cotidiano do professor } \\
\text { de Matemática }\end{array}$ & UFG \\
\hline 1995 & Eleuza de Melo Silva & $\begin{array}{l}\text { O ensino-aprendizagem das operações Ma- } \\
\text { temáticas básicas nas quatro primeiras sé- } \\
\text { ries do ensino fundamental }\end{array}$ & UFG \\
\hline 1997 & Vanda Domingos Vieira & Geometria e Álgebra: uma proposta de ensino & UFG \\
\hline 2000 & $\begin{array}{l}\text { Maria Bethânia Sardeiro } \\
\text { dos Santos }\end{array}$ & $\begin{array}{l}\text { Escrever, para quê? A redação mediando a } \\
\text { formação de conceitos em Cálculo } 1 \text {. }\end{array}$ & UFG \\
\hline 2001 & Adelino Cândido Pimenta & $\begin{array}{l}\text { O Ensino de Funções Lineares numa Abor- } \\
\text { dagem Dinâmica e Iterativa }\end{array}$ & UCG \\
\hline 2001 & Celso de Oliveira Faria & $\begin{array}{l}\text { O computador e a co-construção de conceitos } \\
\text { matemáticos por alunos do ensino fundamen- } \\
\text { tal em uma situação planejada: uma análise } \\
\text { microgenética dos processos de mediação }\end{array}$ & UFG \\
\hline 2001 & Elizabeth Cristina de Faria & $\begin{array}{l}\text { Estudo sobre a contribuição de uma pro- } \\
\text { posta de curso via Internet para a formação } \\
\text { do professor de Matemática }\end{array}$ & UFG \\
\hline 2001 & Vânia Lúcia Machado & $\begin{array}{l}\text { Ser Professor Leigo de Matemática - histó- } \\
\text { ria de vida dos professores }\end{array}$ & UCG \\
\hline 2002 & Suely Vieira Lopes & $\begin{array}{l}\text { Ensino de Matemática: um estudo de caso } \\
\text { de uma prática diferenciada }\end{array}$ & UCG \\
\hline 2003 & $\begin{array}{l}\text { Dagmar Junqueira Gui- } \\
\text { marães da Silva }\end{array}$ & $\begin{array}{l}\text { Os Cursos Superiores de Matemática da } \\
\text { Universidade Católica de Goiás e da Univer- } \\
\text { sidade Federal de Goiás: história e memória }\end{array}$ & UCG \\
\hline 2003 & Enos Pinto da Silva & $\begin{array}{l}\text { Aprendizagem da Matemática - crenças e } \\
\text { obstáculos }\end{array}$ & UEG \\
\hline 2003 & José Carlos Barros da Silva & $\begin{array}{l}\text { Proposta teórico-metodológica para a dis- } \\
\text { ciplina de metodologia do ensino da Ma- } \\
\text { temática baseado no enfoque histórico cul- } \\
\text { tural, na teoria da atividade e na teoria da } \\
\text { formação por etapas das açóes mentais. }\end{array}$ & UEG \\
\hline
\end{tabular}




\section{RevistaSoltaa Voz, v. 17, n. 2}

A pesquisa de Silva (1995) objetivou o desenvolvimento de um estudo sobre o que efetivamente ocorre em sala de aula, durante o processo do ensino e da aprendizagem das operaçōes matemáticas básicas nas quatro primeiras séries do ensino fundamental, partindo de uma pesquisa etnográfica realizada em quatro classes de primeira, segunda, terceira e quarta séries do ensino fundamental de uma escola da rede pública estadual do município de Goiânia, Goiás, durante o primeiro semestre de 1994. A autora indica que a formação acadêmica, intelectual e profissional das professoras não contribuiu para que as mesmas dominassem o que é o processo de elaboração do conhecimento. Isto, segundo Silva (1995), não possibilitou a promoção de um ensino fundamentado na perspectiva interacionista-construtivista e não permitiu que os alunos, por sua vez, desenvolvessem um aprendizado com lógica e com compreensão.

O estudo de Vieira (1997) apresentou sugestões para o processo ensino-aprendizagem da Matemática. Em sua proposta, Vieira valoriza as analogias, as combinações de procedimentos, a generalização de situações como elementos de importância fundamental. Segundo a autora, isto leva o aluno a desenvolver por si próprio a capacidade de discutir conteúdos, propor problemas e resolvê-los, pois assim a aprendizagem da matemática adquirirá um caráter formativo.

A pesquisa de Santos (2000) analisou a formação dos conceitos de derivada, limite e continuidade intermediados pela linguagem escrita (redação) e oral (trabalhos em grupo). O objetivo deste trabalho era "buscar" um ensino de Cálculo $1 \mathrm{em}$ que os alunos se percebessem como pessoas capazes de construírem o seu conhecimento matemático, compreendendo também toda a "lógica" existente que fundamenta estes conteúdos específicos. A pesquisa indicou que o uso da redação nas aulas de Matemática propicia: maior concentração do aprendiz com relação ao seu objeto de estudo; reflexão matemática juntamente com a reflexão sobre seu aprendizado; considerável aprendizagem dos conceitos; apreensão mais efetiva dos conceitos matemáticos; melhoria na capacidade de argumentação, uma maior compreensão da ordem em que foram trabalhados os conteúdos; melhoria na relação professor-aluno, aluno-aluno; espaço para se explorar a criatividade; maior socialização da aprendizagem dos conceitos e percepção de problemas relacionados à auto-estima.

A pesquisa de Celso Faria (2001) pretendeu analisar e discutir o uso do computador pelo professor como ferramenta didática mediadora na 
formação de conceitos, usando a abordagem sociocultural construtivista, que compreende a construção do comportamento por meio das mediações sociais e relações interpessoais. A partir da organização de uma oficina de Matemática onde participaram 10 alunos de $7^{a}$ e $8^{a}$ séries de uma escola da rede particular de ensino de Brasília, pretendeu-se, nessa oficina, criar uma situação-problema que contextualizasse o processo de ensino-aprendizagem dos conceitos de trigonometria e perspectiva, por meio da informática. Entre os resultados obtidos com o desenvolvimento desta oficina. o pesquisador aponta que a relação dinâmica entre alunos, professor, pesquisador, instrutores e o uso de materiais didáticos, destacando-se o computador e os softwares, fez com que os alunos coordenassem os seus próprios interesses e objetivos individuais a fim de alcançarem os objetivos comuns entre os seus parceiros. Isto ocorreu quando as crianças lidaram com os seus conflitos reais.

O estudo de Elizabeth Faria (2001) analisou a viabilidade de uma proposta de formação continuada de professores de Matemática realizada por meio de cursos oferecidos na Internet. Este estudo de caso, em que a pesquisadora organizou e implementou um curso de atualização para professores de Matemática, enfocando o ensino das funções, propiciou a autora a seguinte conclusão: a educação a distância via Internet é algo viável, pois pode contribuir para a formação continuada do professor de Matemática em razão do ambiente interativo que propicia, das condiçôes de pesquisa e de comunicação disponíveis nesse meio.

O trabalho de Machado (2001) objetivou a compreensão do universo, práticas e concepçôes sociais de professores leigos de Matemática, em termos dos saberes que construíram ao longo de suas histórias de vida. Esta se utilizou de relatos orais da "história de vida" de seis professores leigos de Matemática da região do Vale do São Patrício, Goiás, em que se procurou identificar como o professor leigo de Matemática exerce a docência; até que ponto a decisão da carreira profissional sofre influência da formação recebida na sua vida escolar desde os primeiros anos; como esses professores atuam e concebem suas práticas nos diferentes momentos da sua carreira; e, como o professor leigo associa o conteúdo de Matemática à vida cotidiana do aluno. Entre os resultados obtidos, a pesquisadora destaca que o fator sócio-econômico foi determinante na escolha profissional dos professores entrevistados, além disso, colaborou também, para tal escolha, o fato de terem sido bons alunos ou terem professores marcantes. 
170 RevistaSoltaa Voz, v. 17, n. 2

O trabalho de Pimenta (2001) tem como objetivo elaborar uma proposta de ensino para funções lineares baseada no uso do software matemático Linear Web Applet, que associe os conceitos de dinâmica, iterações, gráfico das soluçōes, ponto atrator e repulsor e outras idéias. A partir da realização de uma pesquisa de cunho bibliográfico, Pimenta enfatiza a necessidade de se promoverem ações que consolidem a verticalização do ensino dos conceitos inerentes às funções lineares de forma dinâmica e iterativa, desde o Ensino Fundamental, indicando suas conexões com o Ensino Médio e Superior. Além disso, aponta para a necessidade da incorporação de aplicativos como ferramentas de ensino, pois estes podem promover a mediação que se defende e que se considere necessária ao desenvolvimento da aprendizagem.

O trabalho de Lopes (2002) buscou compreender como ocorre o processo ensino-aprendizagem de Matemática quando norteado por um projeto pedagógico voltado especificamente para a disciplina de Matemática. Esta pesquisa constituiu-se em um estudo de caso, no sentido estrito de estudo descritivo de uma unidade, desenvolvido numa escola da rede particular de ensino em Goiânia. Neste estudo, procurou-se observar a coerência entre os princípios teóricos (piagetianos) que orientam a proposta de ensino na escola e a prática pedagógica desenvolvida pelos professores de Matemática da 5a $6^{\mathrm{a}}$ e $7^{\mathrm{a}}$ séries. Como resultado, Lopes aponta para a possibilidade de se formular uma proposta pedagógica alternativa para o ensino de matemática, desde que os professores e equipe técnica se proponham a estudar e modificar a concepção da matemática a ser ensinada. Além disso, há necessidade de se refletir sobre o como ensinar, de modo que se identifique coerência entre os princípios teóricos que orientam a proposta de ensino da escola e a prática pedagógica desenvolvida pelos professores.

O estudo de Dagmar Silva (2003) buscou compreender e analisar o processo de criação e consolidação de cursos de Matemática da Universidade Católica de Goiás e da Universidade Federal de Goiás, reconstituindo a trajetória histórica de cada um, por meio de documentos escritos. Por meio da história oral, o estudo permite concluir que, embora tenham sido criados em Faculdades de Ciências e Letras, com o objetivo de formar professores para atender à necessidade de professores qualificados para o ensino de Matemática no estado de Goiás, no decorrer dos anos, os dois cursos passaram a apresentar características distintas: o da UCG 
passou a integrar o departamento de Matemática e Física, continuando com o seu ideal de formar professores, ao passo que o da UFG, do então Instituto de Matemática e Física, tem como principal meta a formação de bacharéis, embora atualmente haja uma vertente mais acentuada para a licenciatura.

Por fim, a análise feita das dissertaçooes defendidas no programa da UEG baseou-se na ata de defesa pública destas pesquisas, documento este cedido pela Coordenadora do Curso, pois não conseguimos encontrar uma versão escrita ou eletrônica (nas bibliotecas da instituição ou com os autores) destas dissertações. Desta forma, a análise destas obras ficou comprometida. Apesar disto, elas foram incluídas com o intuito de consolidar o caráter de estado de arte deste trabalho. Neste sentido, somente temos o objetivo do trabalho de Enos Silva (2003) que buscou analisar o ensino-aprendizagem da Matemática sob o foco dos conteúdos trabalhados e os procedimentos didático-pedagógicos utilizados no desenvolvimento da disciplina. Já do trabalho de José Carlos Silva (2003), não foi coletada nenhuma informação, como já foi explicitado.

\section{UMA “Leitura” dos Trabalhos}

A leitura analítica de qualquer pesquisa pode ser feita de várias formas díspares, isto é, fica a critério do pesquisador qual a organização ou categorização a ser seguida. Entre as várias possibilidades, optamos por analisar a produção em Educação Matemática por meio da classificação dos trabalhos a partir de elementos essenciais presentes na pesquisa como: foco temático; metodologia de pesquisa; grau de ensino pesquisado e outros.

O foco temático foi a nossa primeira categoria de organização. Para Fiorentini (2002, p. 4), "essa forma de organização exige que se identifique, para cada trabalho, o foco principal da investigação. Esse processo não é simples ou direto, pois acontece de forma indutiva e, às vezes, dedutiva, exigindo ajustes individuais (para cada estudo) e grupais (envolvendo um conjunto de estudos)".

Porém, a própria escolha das categorias é uma questão complexa, pois elas sofrem alterações ou mudanças de denominações em função de aspectos que passam a ser valorizados em certo período histórico. Para efeitos do nosso estudo, utilizamos as seguintes categorias, que estão apresentadas no quadro 2: concepções e visōes sobre a Matemática e o 
172 RevistaSoltaa Voz, v. 17, n. 2

ensino de Matemática; História da Matemática e História do ensino da Matemática; Didática e ensino da Matemática; Formação de professores de Matemática; e Tecnologias da Informação e da Comunicação e Educação Matemática.

QUADRO 2: As dissertações e os focos temáticos

\begin{tabular}{|l|c|c|}
\hline \multicolumn{1}{|c|}{ Foco temático } & \multicolumn{2}{c|}{ Dissertações } \\
\hline $\begin{array}{l}\text { Concepçóes e visões sobre a Matemática e o Ensino da } \\
\text { Matemática }\end{array}$ & 01 & $8,33 \%$ \\
\hline História da Matemática e a História do Ensino da Matemática & 01 & $8,33 \%$ \\
\hline Didática e Ensino da Matemática & 05 & $41,67 \%$ \\
\hline Formação de professores & 02 & $16,67 \%$ \\
\hline $\begin{array}{l}\text { Tecnologias da informação e da comunicação e a Educação } \\
\text { Matemática }\end{array}$ & 03 & $25,00 \%$ \\
\hline
\end{tabular}

A segunda categoria de organização utilizada por nós refere-se à metodologia de pesquisa utilizada nos estudos analisados. Se a classificação por foco temático já se constitui em uma tarefa delicada, imagine uma análise baseada nas tendências de pesquisa. A complexidade desta questão é apontada por D’Ambrosio (2004, p. 22): "É muito difícil identificar linhas de pesquisa padrão, sobretudo em Educação, e particularmente na pesquisa qualitativa". Contudo, tendo em mente que o nosso objetivo é mostrar as linhas de pesquisa que caracterizem de forma geral os trabalhos, organizamos uma classificação (veja quadro 3), que pode conter imperfeiçôes, mas, no entanto serve ao propósito desta pesquisa. As linhas de pesquisa escolhidas estão todas vinculadas ao viés qualitativo e foram estudo de caso, pesquisa-ação e pesquisa bibliográfica.

QUADRO 3: A metodologia de pesquisa utilizada nas dissertações

\begin{tabular}{|l|c|c|}
\hline \multicolumn{1}{|c|}{ Metodologia de Pesquisa } & \multicolumn{2}{c|}{ Dissertações } \\
\hline Pesquisa bibliográfica & 01 & $8,33 \%$ \\
\hline Pesquisa-ação & 03 & $25,00 \%$ \\
\hline Estudo de caso & 07 & $58,34 \%$ \\
\hline Não informado & 01 & $8,33 \%$ \\
\hline
\end{tabular}

Finalmente, como forma de complementar a "leitura" dos trabalhos, também fizemos uma categorização das dissertaçōes por meio do grau de ensino pesquisado (quadro 4). 
QUADRO 4: O grau de ensino pesquisado nas dissertaçōes

\begin{tabular}{|l|c|c|}
\hline \multicolumn{1}{|c|}{ Grau de ensino pesquisado } & \multicolumn{2}{c|}{ Dissertações } \\
\hline Ensino Fundamental & 04 & $33,34 \%$ \\
\hline Ensino Médio & 01 & $8,33 \%$ \\
\hline Ensino Superior & 05 & $41,67 \%$ \\
\hline Educação Básica & 01 & $8,33 \%$ \\
\hline Não informado & 01 & $8,33 \%$ \\
\hline
\end{tabular}

A análise do teor das pesquisas realizadas nos programas de pósgraduação em Educação sediados nas universidades do Estado de Goiás, durante quinze anos, permitiu identificar uma significativa preocupação com relação às questôes relacionadas à didática e à prática de ensino de Matemática; ao uso das tecnologias da informação e da comunicação e às suas relações com a Educação Matemática.

Além disso, a análise permitiu evidenciar a ausência de trabalhos relacionados a temas como a etnomatemática, a psicologia da Educação Matemática e a avaliação. Adicionalmente, verificamos que são raros os trabalhos que focalizam a História da Matemática e a História do Ensino da Matemática.

\section{CONSIDERAÇŌES FinaIS}

A realização deste levantamento da produção realizada nos programas de pós-graduação do Estado de Goiás sobre a Educação Matemática, no período de 1990 a 2004, possibilitou uma visão geral do estado do conhecimento sobre as questôes relacionadas ao ensino da Matemática. O panorama vislumbrado somente confirmou a análise da pós-graduação realizada pela Capes (2004), que revela a baixa representatividade da produção científica realizada nas instituições de ensino superior situadas em Goiás, com relação à produção nacional. Os 12 trabalhos apresentados aqui correspondem a somente $1,14 \%$ das 1051 teses e dissertações defendidas no Brasil, no período de 1970 até 2003 (Zetetike, 1993-2004).

Desta forma, o grande desafio para a Educação Matemática goiana consiste em estabelecer-se como uma área atuante. A nosso ver um dos caminhos possíveis e mais viáveis para esta consolidação, baseia-se na abertura e criação de linhas de pesquisa destinadas ao ensino da Matemática, dentro dos programas de pós-graduação stricto sensu já estabelecidos no Es- 


\section{RevistaSoltaa Voz, v. 17, n. 2}

tado. Esta iniciativa vai ao encontro das metas estabelecidas pelo Programa Nacional de Pós-graduação (Capes, 2004), que aponta para a necessidade do fortalecimento e crescimento dos programas da região Centro-Oeste.

Finalizando, sugerimos a continuidade e a permanente atualização destes dados, que poderão ampliar o conhecimento sobre a trajetória percorrida, aumentando assim a efetividade dos caminhos a serem trilhados para a garantia do desenvolvimento deste campo de pesquisa dentro do Estado de Goiás.

\section{REFERÊNCIAS}

CAPES. Plano nacional de Pós-graduação (PNPG): 2005-2010. Brasília: Capes, 2004.

Apresenta informaçōes sobre os programas de pós-graduação reconhecidos no Brasil. 2005. Disponível em: <http://www.capes.gov.br>. Acesso em: 12. ago. 2005.

D’AMBrÓSIO, U. Prefácio. In: BORBA, M.; ARAUjO, J. (Orgs.). Pesquisa qualitativa em Educação Matemática. Belo Horizonte: Autêntica, 2004.

Da realidade à ação: reflexões sobre Educação e Matemática. 4. ed. São Paulo: Summus, 1986.

Educação Matemática: da teoria à prática. 10. ed. Campinas, SP: Papirus, 1996 (Coleção Perspectivas em Educação Matemática).

FE-UFG. FACULDADE DE EDUCAÇÃO DA UFG. Apresenta sobre o programa de pós-graduação da instituição. 2005. Disponível em: <http:// www.fe.ufg.br/>. Acesso em: 12. ago. 2005.

FARIA, C. O. O computador e a co-construção de conceitos matemáticos por alunos do ensino fundamental em uma situação planejada: uma análise microgenética dos processos de mediação. 2001. 199 f. Dissertação (Mestrado em Educação Brasileira) - Faculdade de Educação, Universidade Federal de Goiás, Goiânia, 2001.

FARIA, E. C. de. Estudo sobre a contribuiçāo de uma proposta de curso via Internet para a formação do professor de Matemática. 2001. $112 \mathrm{f}$. Dissertação 
(Mestrado em Educação Brasileira) - Faculdade de Educação, Universidade Federal de Goiás, Goiânia, 2001.

FIORENTINI, D. Mapeamento e balanço dos trabalhos do GT-19 (Educação Matemática) no período de 1998 a 2001. In: Anais da 25a Reunião Anual da ANPED. Caxambu/MG, 2002, p. 1-17.

LOPES, S. Ensino de Matemática: um estudo de caso de uma prática diferenciada. 2002. 125 f. Dissertação (Mestrado em Educação) - Universidade Católica de Goiás, Goiânia, 2002.

MACHADO, A.; FONSECA, M. \& GOMES, M. Apresentação: dossiê - a pesquisa em Educação Matemática no Brasil. Educação em revista, Belo Horizonte: FE-UFMG, ano 17, n.36, dez. 2002.

MACHADO, V. Ser professor leigo de Matemática - história de vida dos professores. 2001. 157 f. Dissertação (Mestrado em Educação) - Universidade Católica de Goiás, Goiânia, 2001.

PIMENTA, A. O ensino de Funçôes Lineares numa abordagem dinâmica e Iterativa. 2001. 123 f. Dissertação (Mestrado em Educação) - Universidade Católica de Goiás, Goiânia, 2001.

SANTOS, M. Escrever, para quê? A redação mediando a formação de conceitos em Cálculo 1. 2000. 151 f. Dissertação (Mestrado em Educação Brasileira) - Faculdade de Educação, Universidade Federal de Goiás, Goiânia, 2000 .

SILVA, E. de M. O ensino-aprendizagem das operaçôes matemáticas básicas nas quatro primeiras séries do ensino fundamental. 1995. $224 \mathrm{f}$. Dissertação (Mestrado em Educação Brasileira) - Faculdade de Educação, Universidade Federal de Goiás, Goiânia, 1995.

SILVA, D. J. da. Os cursos superiores de Matemática da Universidade Católica de Goiás e da Universidade Federal de Goiás: história e memória. 2003. 127 f. Dissertação (Mestrado em Educação) - Universidade Católica de Goiás, Goiânia, 2003.

SILVA, E. P. da. Aprendizagem da Matemática - crenças e obstáculos. 2003. [s/f]. Dissertação (Mestrado em Ciências da Educação Superior) - Universidade Estadual de Goiás, Anápolis, 2003. 


\section{RevistaSoltaa Voz, v. 17, n. 2}

SILVA, J. C. B. da. Proposta teórico-metodológica para a disciplina de metodologia do ensino da matemática baseado no enfoque histórico cultural, na teoria da atividade e na teoria da formação por etapas das açôes mentais. 2003. [s/f]. Dissertação (Mestrado em Ciências da Educação Superior) - Universidade Estadual de Goiás, Anápolis, 2003.

UCG. Mestrado em Educação da UCG. Apresenta informações sobre o programa de pós-graduação da instituição. 2005. Disponível em: <http://www. ucg.br/vpg/mestrado/Educacao/dissertacoes.htm\#4a\%20TURMA>. Acesso em: 12. ago. 2005.

UEG. Pró-Reitoria de Pesquisa e Pós-Graduação da UEG (PRPG-UEG). Apresenta informaçōes sobre o programa de pós-graduação da instituição. 2005. Disponível em: <http://www.prp.ueg.br/>. Acesso em: 12. ago. 2005.

VARIZO, Z. História de vida e cotidiano do professor de Matemática. 1990. 285f. Dissertação (Mestrado em Educação Brasileira) - Faculdade de Educação, Universidade Federal de Goiás, Goiânia, 1990.

VIEIRA, V. Geometria e Álgebra: uma proposta de ensino. 1997. $124 \mathrm{f}$. Dissertação (Mestrado em Educação Brasileira) - Faculdade de Educação, Universidade Federal de Goiás, Goiânia, 1997.

ZETETIKE. Revista do CEMPEM da Faculdade de Educação da Unicamp, São Paulo, Semestral, 1993-2004.

Recebido em: 9 set. 2006

Aceito em: 27 nov. 2006 